\title{
NOTES ON SQUARE-INTEGRABLE COHOMOLOGY SPACES ON CERTAIN FOLIATED MANIFOLDS
}

\author{
BY
}

SHINSUKE YOROZU

\begin{abstract}
We discuss some square-integrable cohomology spaces on a foliated manifold with one-dimensional foliation whose leaves are compact and with a complete bundle-like metric. Applications to a contact manifold are given.
\end{abstract}

1. Introduction. On a compact foliated manifold with a bundle-like metric, B. L. Reinhart [9] proved that the cohomology of base-like differential forms is isomorphic to the harmonic space of a certain semidefinite Laplacian. In his paper [4], H. Kitahara discussed the square-integrable basic cohomology spaces on a foliated manifold with a complete bundle-like metric.

In this note, we discuss some square-integrable cohomology spaces on a foliated manifold with one-dimensional foliation whose leaves are compact and with a complete bundle-like metric. Moreover, we give applications to a contact manifold.

The author wishes to express his gratitude to Professor H. Kitahara for several useful suggestions.

2. Preliminaries. We assume that all objects and maps are of class $C^{\infty}$. Let $M$ be a connected, orientable, $(n+1)$-dimensional Riemannian foliated manifold with one-dimensional foliation $\mathscr{F}$ whose leaves are compact. We assume that the Riemannian metric (, ) on $M$ is a bundle-like metric with respect to $\mathscr{F}$ (cf. [8]).

Let $\left\{U ;\left(x, y^{1}, \ldots, y^{n}\right)\right\}$ denote a flat coordinate neighborhood system with respect to $\mathscr{F}$, that is, the integral manifolds of $\mathscr{F}$ are given locally by $y^{1}=c^{1}, \ldots, y^{n}=c^{n}$, for some constants $c^{1}, \ldots, c^{n}$ (cf. [8]). We may choose, in each flat coordinate neighborhood system $\left\{U ;\left(x, y^{1}, \ldots, y^{n}\right)\right\}$, 1 -form $\eta$ and vectors $v_{1}, \ldots, v_{n}$ such that $\left\{\eta, d y^{1}, \ldots, d y^{n}\right\}$ and $\left\{\partial / \partial x, v_{1}, \ldots, v_{n}\right\}$ are dual bases for the cotangent and tangent spaces respectively at each point in $U$. Hence

Received by the editors May 31, 1978 and, in revised form, November 31, 1978.

AMS (MOS) subject classifications (1970). Primary 57D30.

Key words and phrases. Riemannian foliated manifold, bundle-like metric, square-integrable cohomology. 


$$
\begin{aligned}
\eta & =d x+\sum A_{i} d y^{i}, \\
v_{i} & =\partial / \partial y^{i}-A_{i} \partial / \partial x, \quad i=1,2, \ldots, n
\end{aligned}
$$

(cf. [8], [12]). Throughout this note, all local expressions for forms and vectors are taken with respect to these bases.

We may choose, in (1), $A_{i}=A_{i}(x, y)$ such that $\left(\partial / \partial x, v_{i}\right)=0, i=$ $1,2, \ldots, n$, where $y=\left(y^{1}, \ldots, y^{n}\right)$. Then the metric has the local expression

$$
d s^{2}=g_{\Delta \Delta}(x, y) \eta \cdot \eta+\sum g_{i j}(y) d y^{i} \cdot d y^{j},
$$

where $g_{\Delta \Delta}(x, y)=(\partial / \partial x, \partial / \partial x)$ and $g_{i j}(y)=\left(v_{i}, v_{j}\right)$, since the metric $($,$) is a$ bundle-like metric with respect to $\mathscr{F}$ (cf. [8], [12]).

Definition (CF. [8], [12]). A form $\phi$ on $M$ is called of type $(1, s)$ (resp. $(0, s)$ ) if it is expressed locally as

$$
\phi=\frac{1}{1 ! s !} \sum \phi_{\Delta i_{1}} \cdots i_{s}(x, y) \eta \wedge d y^{i_{1}} \wedge \cdots \wedge d y^{i_{n}}
$$

(resp. $\left.\phi=(1 / 0 ! s !) \sum \phi_{i_{1}} \ldots i_{s}(x, y) d y^{i_{1}} \wedge \cdots \wedge d y^{i_{n}}\right)$.

Let $\wedge^{1, s}(M)$ (resp. $\left.\wedge^{0, s}(M)\right)$ be the space of all forms on $M$ of type $(1, s)$ (resp. $(0, s))$. The space $\wedge^{s}(M)$ of all $s$-forms on $M$ is the direct sum of $\wedge^{1, s-1}(M)$ and $\wedge^{0, s}(M)$.

The operator $d$ of exterior differentiation is decomposed as $d=d^{\prime}+d^{\prime \prime}+$ $d^{\prime \prime \prime}$ where the components are of type $(1,0),(0,1)$ and $(-1,2)$ respectively (cf. [9], [12]). Since the foliation $\mathcal{F}$ is of one dimension, we notice the following: (i) If $\phi \in \wedge^{1, s}(M)$, then $d \phi=d^{\prime \prime} \phi+d^{\prime \prime \prime} \phi$, where $d^{\prime \prime} \phi \in$ $\wedge^{1, s+1}(M)$ and $d^{\prime \prime \prime} \phi \in \bigwedge^{0, s+2}(M)$. (ii) If $\phi \in \bigwedge^{0, s}(M)$, then $d \phi=d^{\prime} \phi+$ $d^{\prime \prime} \phi$, where $d^{\prime} \phi \in \bigwedge^{1, s}(M)$ and $d^{\prime \prime} \phi \in \bigwedge^{0, s+1}(M)$.

Among examples we show the following one for reference below:

EXAMPLE. Let $R^{3}$ be a Euclidean space with cartesian coordinates $\left(x, y^{1}, y^{2}\right)$. We put $\eta=d x+\left(-y^{2}\right) d y^{1}$, then $\left\{\eta, d y^{1}, d y^{2}\right\}$ is a base for the cotangent space at each point in $R^{3}$. Let $\xi$ be a dual of $\eta$. Then $R^{3}$ is considered a foliated manifold whose leaves are orbits of vector field $\xi$. We define a metric $d s^{2}$ on $R^{3}$ by

$$
d s^{2}=d x \cdot d x+2\left(-y^{2}\right) d x \cdot d y^{1}+\left(1+\left(y^{2}\right)^{2}\right) d y^{1} \cdot d y^{1}+d y^{2} \cdot d y^{2}
$$

(cf. [10]). Then we have $d s^{2}=\eta \cdot \eta+d y^{1} \cdot d y^{1}+d y^{2} \cdot d y^{2}$. Thus the metric $d s^{2}$ is a complete bundle-like metric with respect to the foliation.

Let $\varphi_{m}: R^{3} \rightarrow R^{3}$ be a map defined by

$$
\varphi_{m}\left(x, y^{1}, y^{2}\right)=\left(x+m,(-1)^{m} y^{1},(-1)^{m} y^{2}\right),
$$

where $m$ is an integer. Define an equivalence relation in $R^{3}$ by $\left(x, y^{1}, y^{2}\right) \sim$ $\left(\tilde{x}, \tilde{y}^{1}, \tilde{y}^{2}\right)$ if there exists an integer $m$ such that $\varphi_{m}\left(x, y^{1}, y^{2}\right)=\left(\tilde{x}, \tilde{y}^{1}, \tilde{y}^{2}\right)$. It is trivial that $\eta$ and $d s^{2}$ are invariant by $\varphi_{m}$ for any $m$. Thus the induced $\eta$ on $M=R^{3} / \sim$ define a foliation on $M$ whose leaves are of one dimension and 
compact, and the induced $d s^{2}$ on $M$ is a complete bundle-like metric with respect to the foliation. We notice that the foliation is not regular (cf. [8]).

3. Spaces $\Delta^{1, *}(M)$ and $\Delta^{0, *}(M)$. Hereafter, we are interested in forms such that

$$
\phi=\frac{1}{1 ! s !} \sum \phi_{\Delta i_{1} \cdots i_{s}}(y) \eta \wedge d y^{i_{1}} \wedge \cdots \wedge d y^{i_{s}}
$$

and

$$
\phi=\frac{1}{0 ! s !} \sum \phi_{i_{1}} \cdots i_{s}(y) d y^{i_{1}} \wedge \cdots \wedge d y^{i_{s}}
$$

Let $\Delta^{1, s}(M)$ (resp. $\left.\Delta^{0, s}(M)\right)$ be the subspace of $\wedge^{1, s}(M)$ (resp. $\wedge^{0, s}(M)$ ) satisfying (I) (resp. (II)) and $\Delta_{0}^{1, s}(M)$ (resp. $\Delta_{0}^{0, s}(M)$ ) the subspace of $\Delta^{1, s}(M)$ (resp. $\left.\Delta^{0, s}(M)\right)$ composed of forms with compact supports.

Proposition 3.1 (CF. [9]). For $\phi \in \bigwedge^{0, s}(M), d^{\prime} \phi=0$ if and only if $\phi \in$ $\Delta^{0, s}(M)$.

A form in $\Delta^{0, s}(M)$ is called a basic or base-like form (cf. [4], [9]).

Restricted to $\Delta^{0, *}(M)=\Sigma \Delta^{0, s}(M), d^{\prime \prime 2}=d^{2}=0$ so we may consider the cohomology of $\Delta^{0, *}(M)$ and $d^{\prime \prime}$ (cf. [9], [12]). Next, if we have an assumption that

$$
A_{i}=A_{i}(y), \quad i=1,2, \ldots, n,
$$

in (1), then we have $d^{\prime \prime 2}=d^{2}=0$ on $\Delta^{1, *}(M)=\Sigma \Delta^{1, s}(M)$ (cf. [12]). The example in $\$ 2$ satisfies our assumption (III). Hereafter, we consider the spaces $\Delta^{1, s}(M)$ and $\Delta^{1, *}(M)$ under the assumption (III). Thus we may consider the cohomology of $\Delta^{1, *}(M)$ and $d^{\prime \prime}$.

4. Square-integrable basic cohomology spaces $\tilde{H}_{2}^{0, s}(M)$. This and the next sections are due to $\mathrm{H}$. Kitahara [4], that is, they are the special cases of Kitahara's results. The methods are analogous to those of A. Andreotti and E. Vesentini [1] and K. Okamoto and H. Ozeki [7].

The *"-operation in $\Delta^{0, s}(M)$ is defined by

$$
\begin{aligned}
* " \phi=\frac{1}{(n-s) ! s !} \sum g^{i_{1} j_{1}} & \cdots g^{i_{j} j_{\delta_{j}}} \delta_{j_{1} \cdots j_{s} k_{1} \cdots k_{n-s}}^{1} \\
& \times \sqrt{\operatorname{det}\left(g_{i j}\right)} \phi_{i_{1} \cdots i_{s}} d y^{k_{1}} \wedge \cdots \wedge d y^{k_{n-s}}
\end{aligned}
$$

where $\left(g^{i j}\right)$ denotes the inverse matrix of $\left(g_{i j}\right)$ and $\delta_{j_{1}}^{1} \cdots j_{j} k_{1} \cdots k_{n-s}$, the Kronecker symbol (cf. [2], [4], [9], [12]). According to B. L. Reinhart [9], we define a pre-Hilbert metric $\langle,\rangle_{1}$ on $\Delta_{0}^{0, s}(M)$ by

$$
\langle\phi, \psi\rangle_{1}=\int_{M} d x \wedge \phi \wedge^{* \prime \prime} \psi
$$


The differential operator $d^{\prime \prime}$ maps $\Delta^{0, s}(M)$ into $\Delta^{0, s+1}(M)$. We define $\tilde{\delta}^{\prime \prime}$ : $\Delta^{0, s}(M) \rightarrow \Delta^{0, s-1}(M)$ by

$$
\tilde{\delta}^{\prime \prime} \phi=(-1)^{n s+n+1} * " d^{\prime \prime} * " \phi .
$$

Let $\tilde{L}_{2}^{0, s}(M)$ be the completion of $\Delta_{0}^{0, s}(M)$ with respect to the inner product $\langle,\rangle_{1}$. We denote by $\tilde{\partial}_{0}$ the restriction of $d^{\prime \prime}$ to $\Delta_{0}^{0, s}(M)$ and by $\tilde{\theta}_{0}$ the restriction of $\tilde{\delta}^{\prime \prime}$ to $\Delta_{0}^{0, s}(M)$. We define $\tilde{\partial}=\left(\tilde{\theta_{0}}\right)^{*}$ and $\tilde{\theta}=(\tilde{\partial})^{*}$, where ()$^{*}$ denotes the adjoint operator of ( ) with respect to the inner product $\langle,\rangle_{1}$. Then $\tilde{\partial}$ (resp. $\tilde{\theta}$ ) is a closed, densely defined operator of $\tilde{L}_{2}^{0, s}(M)$ into $\tilde{L}_{2}^{0, s+1}(M)$ (resp. $\tilde{L}_{2}^{0, s-1}(M)$ ). Let $D_{\tilde{\partial}}^{0, s}$ (resp. $D_{\tilde{\theta}}^{0, s}$ ) be the domain of the operator $\tilde{\partial}$ (resp. $\tilde{\theta})$ in $\tilde{L}_{2}^{0, s}(M)$ and we put

$$
\begin{aligned}
& Z_{\tilde{\partial}}^{0, s}(M)=\left\{\phi \in D_{\tilde{z}}^{0, s} ; \tilde{\partial} \phi=0\right\}, \\
& Z_{\tilde{\theta}}^{0, s}(M)=\left\{\phi \in D_{\tilde{\theta}}^{0, s} ; \tilde{\theta} \phi=0\right\} .
\end{aligned}
$$

Since $\tilde{\partial}$ and $\tilde{\theta}$ are closed operators, $Z_{\tilde{z}}^{0, s}(M)$ and $Z_{\tilde{\theta}}^{0, s}(M)$ are closed in $\tilde{L}_{2}^{0, s}(M)$. Let $B_{\tilde{\partial}}^{0, s}(M)$ (resp. $B_{\tilde{\theta}}^{0, s}(M)$ ) be the closure of $\tilde{\partial}\left(D_{\tilde{\partial}}^{0, s-1}\right)$ (resp. $\left.\tilde{\theta}\left(D_{\tilde{\theta}}^{0, s+1}\right)\right)$.

DEFinition (CF. [4]). $\tilde{H}_{2}^{0, s}(M)=Z_{\tilde{a}}^{0, s}(M) \ominus B_{3}^{0, s}(M)$ is called the square-integrable basic cohomology space, where $\Theta$ denotes the orthogonal complement of $B_{\theta}^{0, s}(M)$.

LEMMA 4.1 (CF. [4]). $\tilde{H}_{2}^{0, s}(M)=Z_{\tilde{\partial}}^{0, s}(M) \cap Z_{\tilde{\theta}}^{0, s}(M)$.

Since $Z_{\tilde{\partial}}^{0, s}(M)$ and $Z_{\tilde{\theta}}^{0, s}(M)$ are closed in $\tilde{L}_{2}^{0, s}(M), \tilde{H}_{2}^{0, s}(M)$ has canonically the structure of a Hilbert space.

The following orthogonal decomposition theorem is proved analogously to

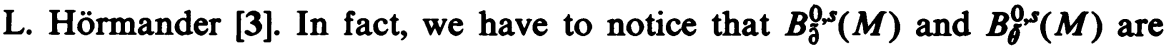
mutually orthogonal and that the intersection of the orthogonal complements of $B_{\vec{\partial}}^{0, s}(M)$ and $B_{\vec{\theta}}^{0, s}(M)$ is $\tilde{H}_{2}^{0, s}(M)$.

THEOREM 4.2 (CF. [4]).

$$
\tilde{L}_{2}^{0, s}(M)=\tilde{H}_{2}^{0, s}(M) \oplus B_{\mathfrak{\partial}}^{0, s}(M) \oplus B_{\tilde{\theta}}^{0, s}(M) .
$$

The following diagram is commutative:

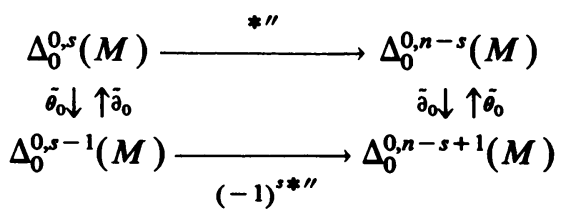

Then we have the Dolbeault-Serre type theorem.

THEOREM 4.3 (CF. [4]). If the bundle-like metric on $M$ is complete, $\tilde{H}_{2}^{0, s}(M)$ $=\tilde{H}_{2}^{0, n-s}(M)$ (isomorphic as Hilbert spaces). 
COROLlaRY 4.4 (CF. [4]). If the bundle-like metric on $M$ is complete and $\operatorname{dim} \tilde{H}_{2}^{0, s}(M)$ is finite, then $\operatorname{dim} \tilde{H}_{2}^{0, s}(M)=\operatorname{dim} \tilde{H}_{2}^{0, n-s}(M)$.

5. $\tilde{\square}$-harmonic forms. In this section, we assume that the bundle-like metric on $M$ is complete.

We consider a function $\mu$ on $\mathbf{R}$ (the reals) satisfying

(i) $0<\mu<1$ on $\mathrm{R}$,

(ii) $\mu(t)=1$ for $t<1$,

(iii) $\mu(t)=0$ for $t>2$.

It is known that a geodesic orthogonal to a leaf is orthogonal to other leaves (cf. [8]). Let $o$ be a point in $M$, and we fix the point $o$. For each point $p$ in $M$, we denote by $\rho(p)$ the distance between leaves through $o$ and $p$. Then we put $w_{k}(p)=\mu(\rho(p) / k), k=1,2,3, \ldots$. We remark that $d^{\prime} w_{k}=0$ and $w_{k} \phi$ has compact support for each $\phi \in \Delta^{0, s}(M)$. We have that $w_{k} \phi \in D_{\tilde{\partial}}^{0, s} \cap D_{\tilde{\theta}}^{0, s}$ for any $\phi \in \Delta^{0, s}(M)$ and

$$
\tilde{\partial}\left(w_{k} \phi\right)=d^{\prime \prime}\left(w_{k} \phi\right), \quad \tilde{\theta}\left(w_{k} \phi\right)=\tilde{\delta^{\prime \prime}}\left(w_{k} \phi\right) .
$$

LEMMA 5.1 (CF. [4]). Under the above notations, there exists a positive number $A$, depending only on $\mu$, such that

$$
\left\|d^{\prime \prime} w_{k} \wedge \phi\right\|^{2}<\left(n A^{2} / k^{2}\right)\|\phi\|^{2}
$$

and

$$
\left\|d^{\prime \prime} w_{k} \wedge^{* \prime \prime} \phi\right\|^{2}<\left(n A^{2} / k^{2}\right)\|\phi\|^{2}
$$

for all $\phi \in \Delta^{0, s}(M)$, where $\|\phi\|^{2}=\langle\phi, \phi\rangle_{1}$.

In order to prove this lemma, we have to notice that the function $\rho$ is a locally Lipschitz function and, at points where the derivatives exist, it holds $\sum g^{i j} v_{i}(\rho) v_{j}(\rho)<n$. Then we have

$$
\left|d^{\prime \prime} w_{k}\right|^{2}=\sum g^{i j} v_{i}\left(w_{k}\right) v_{j}\left(w_{k}\right)<n A^{2} / k^{2},
$$

where $A$ is a positive number depending only on sup $|d \mu / d t|$.

Put

$$
\begin{aligned}
& N_{d^{n}}^{0, s}(M)=\left\{\phi \in \Delta^{0, s}(M) ; d^{\prime \prime} \phi=0\right\} \\
& N_{\delta^{\prime \prime}}^{0, s}(M)=\left\{\phi \in \Delta^{0, s}(M) ; \delta^{\prime \prime} \phi=0\right\}
\end{aligned}
$$

Then we have

Proposition 5.2 (CF. [4]). If the bundle-like metric on $M$ is complete, then

$$
\begin{aligned}
& \left.N_{d^{n}}^{0, s}(M) \cap \tilde{L}_{2}^{0, s}(M) \subset Z_{\partial^{0, s}}^{0, M}\right), \\
& N_{\delta^{, s}}^{0, s}(M) \cap \tilde{L}_{2}^{0, s}(M) \subset Z_{\theta}^{0, s}(M) .
\end{aligned}
$$


Proof. Let $\phi$ be in $N_{d^{\prime, s}}^{0, s}(M) \cap \tilde{L}_{2}^{0, s}(M)$. By (3), we have

$$
\tilde{\partial}\left(w_{k} \phi\right)=d^{\prime \prime}\left(w_{k} \phi\right)=d^{\prime \prime} w_{k} \wedge \phi+w_{k} d^{\prime \prime} \phi=d^{\prime \prime} w_{k} \wedge \phi .
$$

Hence, from Lemma 5.1, we have $\left\|\tilde{\partial}\left(w_{k} \phi\right)\right\|^{2}<\left(n A^{2} / k^{2}\right)\|\phi\|^{2}$. Putting $\phi_{k}=$ $w_{k} \phi$, we have

$$
\tilde{\partial} \phi_{k} \underset{\text { (strong) }}{\rightarrow} 0 \quad(k \rightarrow \infty),
$$

where " $\rightarrow$ (strong)" means "converges strongly to". On the other hand, $\phi_{k}$ $\rightarrow_{\text {(strong) }} \phi(k \rightarrow \infty)$. Since $\tilde{\partial}$ is a closed operator, $\phi$ is in $D_{\tilde{z}}^{0, s}$ and $\tilde{\partial} \phi=0$. This proves $\phi \in Z_{\vec{\partial}}^{0, s}(M)$. In the same way, we may prove the second part.

Definition (CF. [4]). The Laplacian $\tilde{\square}$ acting on $\Delta^{0, *}(M)$ is defined by $\tilde{\square}=d^{\prime \prime} \tilde{\delta}^{\prime \prime}+\tilde{\delta}^{\prime \prime} d^{\prime \prime}$.

Let $B(k)$ be an open tube of radius $k$ of the leaf through the fixed point $o$ in $M$ and $\Delta_{B(k)}^{0, s}(M)$ the space of all forms of type $(0, s)$ with compact support contained in $B(k)$. For $\phi, \psi \in \Delta_{B(k)}^{0, s}(M)$, we put $\langle\phi, \psi\rangle_{B(k)}=\langle\phi, \psi\rangle_{1}$. For any $\phi \in \tilde{L}_{2}^{0, s}(M) \cap \Delta^{0, s}(M)$, we have

$$
\left\langle d^{\prime \prime} \phi, d^{\prime \prime} \alpha\right\rangle_{B(k)}+\left\langle\tilde{\delta}^{\prime \prime} \phi, \tilde{\delta}^{\prime \prime} \alpha\right\rangle_{B(k)}=\langle\tilde{\square} \phi, \alpha\rangle_{B(k)}
$$

for all $\alpha \in \Delta_{B(k)}^{0, s}(M)$. Putting $\alpha=w_{k}^{2} \phi$, we have

$$
\begin{aligned}
& d^{\prime \prime} \alpha=w_{k}^{2} d^{\prime \prime} \phi+2 w_{k} d^{\prime \prime} w_{k} \wedge \phi, \\
& \tilde{\delta}^{\prime \prime} \alpha=w_{k}^{2} \tilde{\delta}^{\prime \prime} \phi+(-1)^{n s+n+1} * "\left(2 w_{k} d^{\prime \prime} w_{k} \wedge * " \phi\right) .
\end{aligned}
$$

Substituting in (4), we have

$$
\begin{aligned}
\left\|w_{k} d^{\prime \prime} \phi\right\|_{B(k)}^{2} & +\left\|w_{k} \tilde{\delta}^{\prime \prime} \phi\right\|_{B(k)}^{2} \\
< & \left|\left\langle\tilde{\square} \phi, w_{k}^{2} \phi\right\rangle_{B(k)}\right|+\left|\left\langle d^{\prime \prime} \phi, 2 w_{k} d^{\prime \prime} w_{k} \wedge \phi\right\rangle_{B(k)}\right| \\
& +\left|\left\langle\tilde{\delta}^{\prime \prime} \phi,{ }^{* \prime \prime}\left(2 w_{k} d^{\prime \prime} w_{k} \wedge{ }^{* \prime \phi} \phi\right)\right\rangle_{B(k)}\right|
\end{aligned}
$$

On the other hand, the Schwarz inequality gives the following

$$
\begin{gathered}
\left|\left\langle d^{\prime \prime} \phi, 2 w_{k} d^{\prime \prime} w_{k} \wedge \phi\right\rangle_{B(k)}\right|<\frac{1}{2}\left(\left\|w_{k} d^{\prime \prime} \phi\right\|_{B(k)}^{2}+4\left\|d^{\prime \prime} w_{k} \wedge \phi\right\|_{B(k)}^{2}\right), \\
\left|\left\langle\tilde{\delta}^{\prime \prime} \phi,{ }^{* \prime \prime}\left(2 w_{k} d^{\prime \prime} w_{k} \wedge^{* \prime \prime} \phi\right)\right\rangle_{B(k)}\right|<\frac{1}{2}\left(\left\|w_{k} \tilde{\delta}^{\prime \prime} \phi\right\|_{B(k)}^{2}+4\left\|d^{\prime \prime} w_{k} \wedge^{* \prime \prime} \phi\right\|_{B(k)}^{2}\right)
\end{gathered}
$$

and

$$
\left|\left\langle\tilde{\square} \phi, w_{k}^{2} \phi\right\rangle_{B(k)}\right|<\frac{1}{2}\left(\frac{1}{\sigma}\left\|w_{k} \phi\right\|_{B(k)}^{2}+\sigma\|\tilde{\square} \phi\|_{B(k)}^{2}\right)
$$

for every $\sigma>0$.

Substituting in (5),

$$
\left\|w_{k} d^{\prime \prime} \phi\right\|_{B(k)}^{2}+\left\|w_{k} \tilde{\delta}^{\prime \prime} \phi\right\|_{B(k)}^{2}<\sigma\|\tilde{\square} \phi\|_{B(k)}^{2}+\left(\frac{1}{\sigma}+\frac{8 n A^{2}}{k^{2}}\right)\|\phi\|_{B(k)}^{2} .
$$

Letting $k \rightarrow \infty$, we have

$$
\left\|d^{\prime \prime} \phi\right\|^{2}+\left\|\tilde{\delta}^{\prime \prime} \phi\right\|^{2}<\sigma\|\tilde{\square} \phi\|^{2}+\frac{1}{\sigma}\|\phi\|^{2}
$$


for every $\sigma>0$. In particular, setting $\tilde{\square} \phi=0$ and letting $\sigma \rightarrow \infty$, we have

LEMMA 5.3 (CF. [4]). Let the bundle-like metric on $M$ be complete. If $\phi \in \tilde{L}_{2}^{0, s}(M) \cap \Delta^{0, s}(M)$ such that $\tilde{\square} \phi=0$, then $d^{\prime \prime} \phi=0$ and $\tilde{\delta}^{\prime \prime} \phi=0$, i.e. $\phi \in N_{d^{n}}^{0, s}(M) \cap N_{\tilde{\delta}^{n}}^{0, s}(M)$.

From Proposition 5.2 and Lemma 5.3, we have the following theorem.

TheOREM 5.4 (CF. [4]). Let the bundle-like metric on $M$ be complete. If $\phi \in \tilde{L}_{2}^{0, s}(M) \cap \Delta^{0, s}(M)$ such that $\tilde{\square} \phi=0$, then $\phi \in \tilde{H}_{2}^{0, s}(M)$.

6. Square-integrable cohomology spaces $H_{2}^{0, s}(M)$ and $H_{2}^{1, s}(M)$. In this section, we set situations under the assumptions

$$
A_{i}=A_{i}(y) \text { and } g_{\Delta \Delta}(x, y)=1
$$

in (1) and (2). The manifold given in the example in $\$ 2$ satisfies (IV).

We notice that the volume element of $M$ is

$$
\begin{aligned}
d V_{M} & =\sqrt{\operatorname{det}\left(\begin{array}{cc}
g_{\Delta \Delta} & 0 \\
0 & g_{i j}
\end{array}\right)} \eta \wedge d y^{1} \wedge \cdots \wedge d y^{n} \\
& =\sqrt{\operatorname{det}\left(g_{i j}\right)} \eta \wedge d y^{1} \wedge \cdots \wedge d y^{n} \quad(\text { from (IV)) } \\
& =\sqrt{\operatorname{det}\left(g_{i j}\right)} d x \wedge d y^{1} \wedge \cdots \wedge d y^{n} \quad \text { (from (1)). }
\end{aligned}
$$

The *-operation on $\Delta^{1, s}(M)$ or $\Delta^{0, s}(M)$ is defined as follows. For $\phi \in$ $\Delta^{1, s}(M)$ and $\psi \in \Delta^{0, s}(M)$,

$$
\begin{aligned}
& { }^{*} \phi=\frac{(-1)^{(1-1) s}}{(1-1) !(n-s) ! 1 ! s !} \sum g^{\Delta \Delta^{i_{1} j_{1}}} \cdots g^{i_{s} j_{s}} \\
& \times \delta_{\Delta j_{1} \cdots j_{s} k_{1} \cdots k_{n-s}}^{\Delta 1 \cdots n} \sqrt{\operatorname{det}\left(\begin{array}{cc}
g_{\Delta \Delta} 0 \\
0 & g_{i j}
\end{array}\right)} \phi_{\Delta i_{1} \cdots i_{s}} d y^{k_{1}} \wedge \cdots \wedge d y^{k_{n-s}} \\
& =\frac{1}{(n-s) ! s !} \sum g^{i_{1} j_{1}} \cdots g^{i j_{j} \delta_{j_{1}}^{1} \cdots j_{j} k_{1} \cdots k_{n-s}} \\
& \times \sqrt{\operatorname{det}\left(g_{i j}\right)} \phi_{\Delta i_{1} \cdots i_{s}} d y^{k_{1}} \wedge \cdots \wedge d y^{k_{n-s}}, \\
& * \psi=\frac{(-1)^{(1-0) s}}{(1-0) !(n-s) ! 0 ! s !} \sum g^{i_{1} j_{1}} \cdots g^{i_{3} j_{j}} \delta_{j_{1}}^{1} \cdots j_{j} k_{1} \cdots k_{n-s} \\
& \times \sqrt{\operatorname{det}\left(\begin{array}{cc}
g_{\Delta \Delta} & 0 \\
0 & g_{i j}
\end{array}\right)} \psi_{i_{1} \cdots i_{3}} \eta \wedge d y^{k_{1}} \wedge \cdots \wedge d y^{k_{n-1}} \\
& =\frac{(-1)^{s}}{(n-s) ! s !} \sum g^{i_{1} j_{1}} \cdots g^{i j_{j} \delta_{j_{1}}^{1} \cdots j_{j} k_{1} \cdots k_{n-s}} \\
& \times \sqrt{\operatorname{det}\left(g_{i j}\right)} \psi_{i_{1}} \ldots i_{j} \eta \wedge d y^{k_{1}} \wedge \cdots \wedge d y^{k_{n-s}}
\end{aligned}
$$


(cf. [2], [12]). The operator ${ }^{*}$ maps $\Delta^{1, s}(M)\left(\right.$ resp. $\left.\Delta^{0, s}(M)\right)$ into $\Delta^{0, n-s}(M)$ (resp. $\left.\Delta^{1, n-s}(M)\right)$, since (IV) holds.

We define a pre-Hilbert metric on $\Delta_{0}^{1, s}(M)\left(\right.$ or $\left.\Delta_{0}^{0, s}(M)\right)$ by $\langle\phi, \psi\rangle=\int_{M} \phi$ $\wedge^{*} \psi$. The differential operator $d^{\prime \prime}$ maps $\Delta^{1, s}(M)$ (resp. $\Delta^{0, s}(M)$ ) into $\Delta^{1, s+1}(M)$ (resp. $\left.\Delta^{0, s+1}(M)\right)$. We define $\delta^{\prime \prime}: \Delta^{1, s}(M) \rightarrow \Delta^{1, s-1}(M)$ (or $\Delta^{0, s}(M)$ $\left.\rightarrow \Delta^{0, s-1}(M)\right)$ by

$$
\begin{aligned}
\delta^{\prime \prime} \phi & =(-1)^{(n+1)(s+1)+(n+1)+1} * d^{\prime \prime} * \phi \\
& =(-1)^{n s+s+1 * d^{\prime \prime} * \phi,} \\
\delta^{\prime \prime} \psi & =(-1)^{(n+1) s+(n+1)+1 * d^{\prime \prime} * \psi} \\
& =(-1)^{n s+s+n *} d^{\prime \prime} * \psi
\end{aligned}
$$

for $\phi \in \Delta^{1, s}(M)$ and $\psi \in \Delta^{0, s}(M)$. Then we have $\left\langle d^{\prime \prime} \phi, \psi\right\rangle=\left\langle\phi, \delta^{\prime \prime} \psi\right\rangle$ for $\phi \in \Delta_{0}^{1, s}(M)\left(\operatorname{resp} . \Delta_{0}^{0, s}(M)\right)$ and $\psi \in \Delta_{0}^{1, s+1}(M)$ (resp. $\left.\Delta_{0}^{0, s+1}(M)\right)$.

Let $L_{2}^{1, s}(M)$ (resp. $L_{2}^{0, s}(M)$ ) be the completion of $\Delta_{0}^{1, s}(M)$ (resp. $\Delta_{0}^{0, s}(M)$ ) with respect to the inner product $\langle$,$\rangle . We denote by \partial_{0}$ the restriction of $d^{\prime \prime}$ to $\Delta_{0}^{1, s}(M)\left(\right.$ or $\left.\Delta_{0}^{0, s}(M)\right)$ and by $\theta_{0}$ the restriction of $\delta^{\prime \prime}$ to $\Delta_{0}^{1, s}(M)$ (or $\Delta_{0}^{0, s}(M)$ ). Define $\partial=\left(\theta_{0}\right)^{*}$ and $\theta=(\partial)^{*}$ where ()$^{*}$ denotes the adjoint operator of () with respect to the inner product $\langle$,$\rangle . Then \partial$ is a closed, densely defined operator of $L_{2}^{1, s}(M)$ (resp. $\left.L_{2}^{0, s}(M)\right)$ into $L_{2}^{1, s+1}(M)$ (resp. $\left.L_{2}^{0, s+1}(M)\right)$, and $\theta$ is a closed, densely defined operator of $L_{2}^{1, s}(M)$ (resp. $\left.L_{2}^{0, s}(M)\right)$ into $L_{2}^{1, s-1}(M)$ (resp. $L_{2}^{0, s-1}(M)$ ).

The following objects are defined by the same ways as in $\$ 4$ :

$$
\begin{array}{llll}
D_{\partial}^{1, s}, & D_{\partial}^{0, s}, & D_{\theta}^{1, s}, & D_{\theta}^{0, s}, \\
Z_{\partial}^{1, s}(M), & Z_{\partial}^{0, s}(M), & Z_{\theta}^{1, s}(M), & Z_{\theta}^{0, s}(M), \\
B_{\partial}^{1, s}(M), & B_{\partial}^{0, s}(M), & B_{\theta}^{1, s}(M), & B_{\theta}^{0, s}(M) .
\end{array}
$$

Then

DEFINITION. $H_{2}^{1, s}(M)=Z_{\partial}^{1, s}(M) \ominus B_{\partial}^{1, s}(M)$ and $H_{2}^{0, s}(M)=Z_{\partial}^{0, s}(M) \ominus$ $B_{\partial}^{0, s}(M)$.

By the same ways as in $\$ 4$, we have

LEMMA 6.1. Under the assumption (IV),

$$
H_{2}^{1, s}(M)=Z_{\partial}^{1, s}(M) \cap Z_{\theta}^{1, s}(M)
$$

and

$$
H_{2}^{0, s}(M)=Z_{\partial}^{0, s}(M) \cap Z_{\theta}^{0, s}(M) .
$$

THEOREM 6.2. Under the assumption (IV),

$$
L_{2}^{1, s}(M)=H_{2}^{1, s}(M) \oplus B_{\partial}^{1, s}(M) \oplus B_{\theta}^{1, s}(M)
$$

and

$$
L_{2}^{0, s}(M)=H_{2}^{0, s}(M) \oplus B_{\partial}^{0, s}(M) \oplus B_{\theta}^{0, s}(M)
$$


THEOREM 6.3. Under the assumption (IV), if the bundle-like metric on $M$ is complete, then $\mathrm{H}_{2}^{0, s}(M)=H_{2}^{1, n-s}(M)$ (isomorphic as Hilbert spaces).

In order to prove Theorem 6.3 , we have to notice that $\langle\phi, \psi\rangle=\left\langle{ }^{*} \phi,{ }^{*} \psi\right\rangle$ for $\phi, \psi \in \Delta_{0}^{0, s}(M)$.

COROLlaRY 6.4. Under the assumption (IV), if the bundle-like metric on $M$ is complete and $\operatorname{dim} H_{2}^{0, s}(M)$ is finite, then $\operatorname{dim} H_{2}^{0, s}(M)=\operatorname{dim} H_{2}^{1, n-s}(M)$.

Now, we have $\langle\phi, \psi\rangle=\langle\eta \wedge \phi, \eta \wedge \psi\rangle$ for $\phi, \psi \in \Delta_{0}^{0, s}(M)$. Let $\xi$ denote the dual to $\eta$ and $i_{\xi}$ the interior product by $\xi$ operator. Then we have $i_{\xi} \phi \in \Delta^{0, s}(M)$ and $\eta \wedge i_{\xi} \phi=\phi$ for $\phi \in \Delta^{1, s}(M)$. The following diagram is commutative.

$$
\begin{array}{ccc}
\Delta_{0}^{0, s}(M) & \stackrel{e(\eta)}{\rightarrow} & \Delta_{0}^{1, s}(M) \\
\theta_{0} \downarrow \uparrow \partial_{0} & & \partial_{0} \uparrow \downarrow \theta_{0} \\
\Delta_{0}^{0, s-1}(M) & \stackrel{(-1) e(\eta)}{\rightarrow} & \Delta_{0}^{1, s-1}(M)
\end{array}
$$

where $e(\eta)$ denotes the exterior product by $\eta$ operator. Thus we have

TheOREM 6.5. Under the assumption (IV), if the bundle-like metric on $M$ is complete, then $H_{2}^{0, s}(M)=H_{2}^{1, s}(M)$ (isomorphic as Hilbert spaces).

From Theorems 6.3 and 6.5, we have

THEOREM 6.6. Under the assumption (IV), if the bundle-like metric on $M$ is complete, then $H_{2}^{1, s}(M)=H_{2}^{1, n-s}(M)$ and $H_{2}^{0, s}(M)=H_{2}^{0, n-s}(M)$ (isomorphic as Hilbert spaces).

Next, from (IV), it is easy to see that $\langle\phi, \psi\rangle_{1}=\langle\phi, \psi\rangle$ for $\phi, \psi \in \Delta_{0}^{0, s}(M)$. The following diagram is commutative.

$$
\begin{array}{ccc}
\Delta_{0}^{0, s}(M) & \rightarrow & \Delta_{0}^{0, s}(M) \\
\tilde{\theta}_{0} \downarrow \uparrow \tilde{\partial}_{0} & & \partial_{0} \uparrow \downarrow \theta_{0} \\
\Delta_{0}^{0, s-1}(M) & \rightarrow & \Delta_{0}^{0, s-1}(M)
\end{array}
$$

where $I$ denotes the identity map. Thus we have the following theorem.

THEOREM 6.7. Under the assumption (IV), if the bundle-like metric on $M$ is complete, then $\tilde{H}_{2}^{0, s}(M)=H_{2}^{0, s}(M)$ (isomorphic as Hilbert spaces).

7. $\square$-harmonic forms. In this section, we assume that the assumption (IV) holds and that the bundle-like metric on $M$ is complete. We put

$$
\begin{aligned}
& N_{d^{n}}^{1, s}(M)=\left\{\phi \in \Delta^{1, s}(M) ; d^{\prime \prime} \phi=0\right\}, \\
& N_{\delta^{n}}^{1, s}(M)=\left\{\phi \in \Delta^{1, s}(M) ; \delta^{\prime \prime} \phi=0\right\}, \\
& N_{d^{n}}^{0, s}(M)=\left\{\phi \in \Delta^{0, s}(M) ; d^{\prime \prime} \phi=0\right\}, \\
& N_{\delta^{n}}^{0, s}(M)=\left\{\phi \in \Delta^{0, s}(M) ; \delta^{\prime \prime} \phi=0\right\} .
\end{aligned}
$$


Then, by the same ways as in $\S 5$, we have

Proposition 7.1. Let the assumption (IV) hold and the bundle-like metric on $M$ be complete. Then

$$
\begin{array}{ll}
N_{d^{\prime \prime}}^{1, s}(M) \cap L_{2}^{1, s}(M) \subset Z_{\partial}^{1, s}(M), & N_{\delta^{\prime \prime}}^{1, s}(M) \cap L_{2}^{1, s}(M) \subset Z_{\theta}^{1, s}(M), \\
N_{d^{n}}^{0, s}(M) \cap L_{2}^{0, s}(M) \subset Z_{\partial}^{0, s}(M), & N_{\delta^{n}}^{0, s}(M) \cap L_{2}^{0, s}(M) \subset Z_{\theta}^{0, s}(M) .
\end{array}
$$

Definition. The Laplacian acting on $\Delta^{1, *}(M)$ (or $\left.\Delta^{0, *}(M)\right)$ is defined by $\square=d^{\prime \prime} \delta^{\prime \prime}+\delta^{\prime \prime} d^{\prime \prime}$.

By the same ways as in $\$ 5$, we have

LEMMA 7.2. Let the assumption (IV) hold and the bundle-like metric on $M$ be complete. If $\phi \in L_{2}^{1, s}(M) \cap \Delta^{1, s}(M)$ (resp. $\left.L_{2}^{0, s}(M) \cap \Delta^{0, s}(M)\right)$ such that $\square \phi=$ 0 , then $d^{\prime \prime} \phi=0$ and $\delta^{\prime \prime} \phi=0$, i.e. $\phi \in N_{d^{n}}^{0, s}(M) \cap N_{\delta^{n}}^{1, s}(M)$ (resp. $N_{d^{n}}^{0, s}(M) \cap$ $\left.N_{\delta " ~}^{0, s}(M)\right)$.

From Proposition 7.1 and Lemma 7.2, we have

THEOREM 7.3. Let the assumption (IV) hold and the bundle-like metric on $M$ be complete. If $\phi \in L_{2}^{1, s}(M) \cap \Delta^{1, s}(M)$ (resp. $L_{2}^{0, s}(M) \cap \Delta^{0, s}(M)$ ) such that $\square \phi=0$, then $\phi \in H_{2}^{1, s}(M)$ (resp. $\left.H_{2}^{0, s}(M)\right)$.

From Theorems 5.4 and 6.7, we have

THEOREM 7.4. Let the assumption (IV) hold and the bundle-like metric on $M$ be complete. If $\phi \in \tilde{L}_{2}^{0, s}(M) \cap \Delta^{0, s}(M)$ such that $\tilde{\square} \phi=0$, then $\phi \in H_{2}^{0, s}(M)$.

From Theorems 6.7 and 7.3, we have

THEOREM 7.5. Let the assumption (IV) hold and the bundle-like metric on $M$ be complete. If $\phi \in L_{2}^{0, s}(M) \cap \Delta^{0, s}(M)$ such that $\square \phi=0$, then $\phi \in \tilde{H}_{2}^{0, s}(M)$.

REMARK. I. Vaisman [12], [13] already noticed that on a compact orientable Riemannian foliated manifold $M$, the space $\mathcal{H}^{r, s}(M)$ of foliated harmonic forms is a subspace of the de Rham cohomology space $H^{r, s}(M)$.

REMARK. For the relations between certain cohomology spaces and the existence of bundle-like metrics, see H. Kitahara and S. Yorozu [5].

8. Applications to a contact manifold. First, we cite the definition of the contact manifold. A 1-form $\eta$ on a connected $(2 n+1)$-dimensional manifold is called a contact form if $\eta \wedge(d \eta)^{n} \neq 0$ at each point in the manifold (cf. [10]). A connected $(2 n+1)$-dimensional manifold with a contact form is called a contact manifold. On a contact manifold with a contact form $\eta$, there exists a global vector field $\xi$ such that $\eta(\xi)=1$ and $i_{\xi} d \eta=0$ (cf. [10]). A connected paracompact contact manifold with a contact form $\eta$ has a Riemannian metric (, ) such that 


$$
\eta(X)=(X, \xi)
$$

for any vector field $X$ (cf. [10]). In fact, let (, )' be an arbitrary Riemannian metric, and we define

$$
(X, Y)=(X-\eta(X) \xi, Y-\eta(Y) \xi)^{\prime}+\eta(X) \cdot \eta(Y)
$$

for any vector fields $X$ and $Y$. Such a metric (, ) satisfies (6).

Now, let $N$ be a connected $(2 n+1)$-dimensional contact manifold with a contact form $\eta$ and a Riemannian metric (, ) satisfying (6). We assume that $\xi$ is a Killing vector field on $N$ with respect to the metric ( , ) and that the orbits of $\xi$ are compact. An example of such a manifold $N$ is the manifold given in the example in $\$ 2$.

We define the operators $\delta, e(\eta), i_{\xi}, L$ and $\Lambda$ on $\Lambda^{s}(N)$ as follows:

$$
\begin{gathered}
\delta \phi=(-1)^{*} d^{*} \phi, \quad e(\eta) \phi=\eta \wedge \phi, \\
i_{\xi} \phi=(-1)^{s-1} * e(\eta) * \phi, \\
L \phi=d \eta \wedge \phi, \quad \Lambda \phi={ }^{*} L^{*} \phi
\end{gathered}
$$

(cf. [2], [6], [11]).

Definition. A form $\phi$ in $\Lambda^{s}(N)$ is called a $C$-harmonic form (resp. $C^{*}$-harmonic form) if $i_{\xi} \phi=0, d \phi=0$ and $\delta \phi=e(\eta) \Lambda \phi$ (resp. $e(\eta) \phi=0$, $d \phi=i_{\xi} L \phi$ and $\left.\delta \phi=0\right)$.

REMARK. The notion of $C$-harmonic forms was introduced by $\mathrm{S}$. Tachibana [11], and Y. Ogawa [6] gave the definition of $C^{*}$-harmonic forms. They discussed it on compact normal contact metric manifolds. A normal contact metric manifold is a so-called Sasakian manifold (for the definition, see [6], [10], [11]).

For each point in $N$, there exists a local coordinate neighborhood system $\left\{U ;\left(x, y^{1}, \ldots, y^{n}, y^{n+1}, \ldots, y^{2 n}\right)\right\}$ such that

$$
\eta=d x+\sum\left(-y^{n+i}\right) d y^{i} \quad(i=1,2, \ldots, n)
$$

and the orbits of $\xi$ are given locally by

$$
y^{1}=c^{1}, \ldots, y^{n}=c^{n}, y^{n+1}=c^{n+1}, \ldots, y^{2 n}=c^{2 n}
$$

for the same constants $c^{1}, \ldots, c^{n}, c^{n+1}, \ldots, c^{2 n}$ (cf. [10]). $\left\{\eta, d y^{1}, \ldots, d y^{n}\right.$, $\left.d y^{n+1}, \ldots, d y^{2 n}\right\}$ and $\left\{\partial / \partial x, v_{1}, \ldots, v_{n}, v_{n+1}, \ldots, v_{2 n}\right\}$ are dual bases for the cotangent and tangent spaces respectively at each point in $U$, where

$$
v_{i}=\partial / \partial y^{i}+\left(y^{n+i}\right) \partial / \partial x \text { and } v_{n+i}=\partial / \partial y^{n+i} \text {. }
$$

We may consider $N$ as a foliated manifold whose leaves are orbits of $\xi$. From (6), we have

$$
\begin{aligned}
\left(v_{i}, \xi\right) & =\eta\left(v_{i}\right)=0, \\
\left(v_{n+i}, \xi\right) & =\eta\left(v_{n+i}\right)=0 .
\end{aligned}
$$


Then, since $\xi$ is a Killing vector field on $N$, the metric (, ) on $N$ is a bundle-like metric with respect to the foliation, that is, the local expression of the metric (, ) in $U$ is

$$
d s^{2}=\eta \cdot \eta+\sum g_{A B} d y^{A} \cdot d y^{B}
$$

where $A, B=1,2, \ldots, 2 n$. Thus the contact manifold $N$ is a Riemannian foliated manifold with one-dimensional foliation $\mathscr{F}$ whose leaves are compact and the Riemannian metric (, ) on $N$ is a bundle-like metric with respect to $\mathscr{F}$. Moreover, the assumption (IV) in $\$ 6$ is satisfied. Therefore, we may apply the discussions of above sections to the contact manifold $N$.

In order to obtain the applications to $N$, we have to prepare the decomposition of the operator $\delta$. We have the decomposition of the operator $d$ :

$$
d=d^{\prime}+d^{\prime \prime}+d^{\prime \prime \prime}
$$

(cf. §2). Then, according to I. Vaisman [12], we define the operators $\delta^{\prime}, \delta^{\prime \prime}$ and $\delta^{\prime \prime \prime}$ as follows:

$$
\begin{aligned}
\delta^{\prime} \phi & =(-1)^{r+s} * d^{\prime *} \phi, \\
\delta^{\prime \prime} \phi & =(-1)^{r+s} * d^{\prime \prime} * \phi, \\
\delta^{\prime \prime \prime} \phi & =(-1)^{r+s} * d^{\prime \prime \prime} * \phi,
\end{aligned}
$$

where $\phi \in \bigwedge^{r, s}(N), r=0$ or 1 . Then we have the decomposition of the operator $\delta$ :

$$
\delta=\delta^{\prime}+\delta^{\prime \prime}+\delta^{\prime \prime \prime} .
$$

We notice the following: (i) If $\phi \in \bigwedge^{1, s}(N)$, then $\delta \phi=\delta^{\prime} \phi+\delta^{\prime \prime} \phi$, where $\delta^{\prime} \phi \in \bigwedge^{0, s}(N)$ and $\delta^{\prime \prime} \phi \in \bigwedge^{1, s-1}(N)$. (ii) If $\phi \in \bigwedge^{0, s}(N)$, then $\delta \phi=\delta^{\prime \prime} \phi+$ $\delta^{\prime \prime \prime} \phi$, where $\delta^{\prime \prime} \phi \in \bigwedge^{0, s-1}(N)$ and $\delta^{\prime \prime \prime} \phi \in \wedge^{1, s-2}(N)$.

We have easily the following lemma.

LEMMA 8.1. For $\phi \in \Delta^{1, s}(N)$ and $\psi \in \Delta^{0, s}(N)$,

$$
d^{\prime \prime \prime} \phi=i_{\xi} L \phi, \quad \delta^{\prime \prime \prime} \psi=e(\eta) \Lambda \psi \text {. }
$$

From Theorem 7.4 and Lemma 8.1, we have

THEOREM 8.2. Let the metric (, ) on $N$ be complete. If $\phi \in \tilde{L}_{2}^{0, s}(N) \cap$ $\Delta^{0, s}(N)$ such that $\tilde{\square} \phi=0$, then $\phi$ is a C-harmonic form.

From Theorem 7.3 and Lemma 8.1, we have

TheOREM 8.3. Let the metric (, ) on $N$ be complete. If $\phi \in L_{2}^{1, s}(N) \cap$ $\Delta^{1, s}(N)$ such that $\square \phi=0$, then $\phi$ is a $C^{*}$-harmonic form.

Acknowledgement. The author wishes to thank the referee for his valuable suggestions. 


\section{REFERENCES}

1. A. Andreotti and E. Vesentini, Carleman estimates for the Laplace-Beltrami equation on complex manifold, Inst. Hautes Études Sci. Publ. Math. 25 (1965), 313-362. MR 30 \# 5333.

2. S. I. Goldberg, Curvature and homology, Academic Press, New York, 1962. MR 25 \#2537.

3. L. Hörmander, $L^{2}$-estimates and existence theorems for the j-operator, Acta Math. 113 (1965), 89-152. MR 31 \#3691.

4. H. Kitahara, Remarks on square-integrable basic cohomology spaces on a foliated Riemannian manifold, Kodai Math. J. 2 (1979).

5. H. Kitahara and S. Yorozu, On the cohomology groups of a manifold with a nonintegrable subbundle, Proc. Amer. Math. Soc. 59 (1976), 201-204. MR 55 \# 11271.

6. Y. Ogawa, On C-harmonic forms in a compact Sasakian space, Tôhoku Math. J. 19 (1967), 267-296. MR 36 \#4484.

7. K. Okamoto and H. Ozeki, On square-integrable $\bar{\partial}$-cohomology spaces attached to hermitian symmetric spaces, Osaka J. Math. 4 (1967), 95-110. MR 37 \# 4834.

8. B. L. Reinhart, Foliated manifolds with bundle-like metrics, Ann. of Math. (2) 69 (1959), 119-131. MR 21 \#6004.

9. , Harmonic integrals on foliated manifolds, Amer. J. Math. 81 (1959), 529-536. MR 21 \#6005.

10. S. Sasaki, Almost contact manifolds, Part I, Math. Inst. Tôhoku Univ. 1965; ibid., Part II, 1967; ibid., Part III, 1968.

11. S. Tachibana, On a decompasition of C-harmonic forms in a compact Sasakian space, Tôhoku Math. J. 19 (1967), 198-212. MR 36 \#3379.

12. I. Vaisman, Variétés riemanniennes feuilletées, Czechoslovak Math. J. 21 (1971), 46-75. MR 44 \# 4776.

13. Cohomology and differential forms, Marcel Dekker, New York, 1973. MR 49 \#6095.

Department of Mathrmatics, College of liberal Arts, Kanazawa University, KANAZAWA 920, JAPAN 